\title{
Assuring safety and efficacy of nurse triage for electronic consultation to improve access to specialty care
}

\author{
Elizabeth J Murphy, ${ }^{1,2}$ Delphine S Tuot (i) ${ }^{2,3}$
}

${ }^{1}$ Division of Endocrinology at Zuckerberg San Francisco General Hospital, University of California San Francisco, San Francisco, California, USA

${ }^{2}$ Center for Innovation in Access and Quality at Zuckerberg San Francisco General Hospital, University of California San Francisco, San Francisco, California, USA

${ }^{3}$ Division of Nephrology at Zuckerberg San Francisco General Hospital, University of California San Francisco, San Francisco, California, USA

\section{Correspondence to} Dr Delphine S Tuot, Division of Nephrology at Zuckerberg San Francisco General Hospital, University of California San Francisco, San Francisco, CA 94110, USA

delphine.tuot@ucsf.edu

Accepted 23 January 2021 Published Online First 4 February 2021

\section{Sinked}

- http://dx.doi.org/10.1136/ bmjqs-2020-011817

\section{Check for updates}

(c) Author(s) (or their employer(s)) 2021. No commercial re-use. See rights and permissions. Published by BMJ.

To cite: Murphy EJ, Tuot DS. BMJ Qual Saf

2021;30:533-535.
Ensuring delivery of timely, coordinated, safe and cost-effective specialty care is one of the most pressing issues facing our healthcare systems worldwide. ${ }^{1}$ Poor access to specialty care is common across health delivery organisations, with wait times as high as 6-12 months in some communities. $^{2-4}$ For many specialties this is rooted in a shortage of specialist physicians. ${ }^{5}$ A key question, therefore, is how to maximise the efficiency and effectiveness of specialist use and access. The traditional primary-specialty care interface consists of a primary care provider (PCP) faxing a referral request to a specialist's office without much accompanying diagnostic information and waiting to hear from the patient when an appointment has been scheduled (often on a firstcome, first-served basis). The process is inefficient as it often results in avoidable specialist visits, duplicate diagnostic testing and delayed diagnoses for those patients who need specialist evaluation sooner than others who were referred to the same specialist. ${ }^{6-8}$ Newer models for improving access to specialty care are thus urgently needed.

Electronic consultation (eConsult), which provides a way for PCPs and their patients to obtain specialty expertise without an inperson patient visit to the specialist, has emerged as one potential solution for increasing access to specialty care through improved efficiency of consultation. ${ }^{1}$ eConsults are asynchronous virtual provider-to-provider consultations. They allow specialists to address consultative needs through chart review and communication with the referring provider, often without an inperson specialty care visit. They can be used to triage whether an inperson visit is needed, and when such a visit is needed, ensure adequate diagnostic work-up is completed before the visit. Ideally, this results in time saving for scarce specialists and improved wait times for patients.

Studies of early adopters of eConsult programmes in North America, for instance, have demonstrated benefits consistent with the overarching goal of achieving the quadruple aim: high-quality care delivery that improves population health and achieves high satisfaction among providers, care team members and patients, while introducing costsavings from new efficiencies. ${ }^{19}$ Some early adopters have sought to continue to improve the efficiency of eConsults, leveraging advanced practice providers (APP) or registered nurses (RN) to participate in the triage and/or electronic consultation aspects of eConsult programmes under supervision of specialty physicians.

In this issue of BMJ Quality \& Safety, Donovan et $a l^{10}$ describe an intervention that introduces an $\mathrm{RN}$ into the existing eConsult process to sleep medicine specialists. The nurse used a decision support tool based on obstructive sleep apnoea (OSA) guidelines to perform an initial review of referrals for OSA testing in a single Veterans Affairs Medical Center. The nurse triaged the eConsults to one of three options: home sleep testing, inpatient sleep testing with polysomnography or further review by a sleep medicine specialist to determine the optimal testing approach. Home sleep studies are more comfortable and convenient for the patient and are less costly than inpatient sleep testing, but collect less data than inpatient sleep tests and are thus not suitable for individuals with severe chronic obstructive pulmonary disease or comorbid conditions such as advanced congestive heart failure, neuromuscular diseases and cognitive impairment due to the potential for missed OSA diagnoses. 
The authors examined the impact of the $\mathrm{RN}$ triage model versus the traditional specialist triage model on safety of sleep testing (primary outcome), defined as the receipt of home sleep testing in the presence of a contraindication which could lead to a missed diagnosis of OSA. Patients were assigned to be triaged by the $\mathrm{RN}$ or the specialist based on triager availability at the time of referral, making this a quasi-random assignment and stronger study design to assess the safety of nurses independently making these initial triage decisions compared with a cohort study in which the nurse or the specialist selected the eConsults to triage. Study results indicate that patients whose referrals were initially triaged by nurses had a lower prevalence of receiving contraindicated at-home testing compared with patients whose referrals were initially triaged by specialist physicians ( $1.8 \%$ vs $3.3 \%)$. Of the 793 referrals reviewed by a nurse, $62.4 \%$ were sent for further review by a specialist who ultimately made the final triage decision. The high incidence of this secondary triage likely biased the results to the null, making the differences in this safety outcome all the more notable. However, the outcome was not based directly on the triage decision but rather on the sleep tests that were scheduled and completed. The scheduling process after the triage decision was not the same for the traditional specialist-led and nurse-led model, potentially introducing bias into the results. If there was minimal bias and the results were mainly affected by triage decisions, the authors speculate that greater receipt of inappropriate home testing after initial triage by specialists was perhaps because they were less likely to review and follow the local sleep testing recommendations (adapted from the 2017 American Academy of Sleep Medicine guidelines) compared with nurses. As noted, another explanation could stem from differences in the scheduling process after clinician triage.

Of the initial nurse-triaged referrals, 38.6\% (308) were managed independently by the nurse without specialist review. As a secondary analysis, those referrals were compared with referrals triaged by specialists in a propensity matched subsample. In this group, focused on patients for whom the nurse made the triage decision, data suggest there was no difference in prevalence of triage to a contraindicated home sleep test (adjusted relative risk $0.91,95 \%$ CI 0.41 to 1.98 ), supporting the primary findings that there was no safety concern associated with $\mathrm{RN}$ triage decisions.

These 'real world' data are consistent with prior trial literature examining different models of ambulatory care delivery for sleep disordered breathing. For example, in one randomised controlled trial, a protocolised, RN-supervised, home-based diagnosis and treatment intervention was deemed non-inferior to a traditional specialist-led, hospital-based diagnosis and treatment strategy with respect to changes in scores on the Epworth Sleepiness Scale after 3 months. ${ }^{11}$
Although promising, these results must be considered alongside important practical considerations. First, over $60 \%$ of the $\mathrm{RN}$-reviewed referrals were sent for further review by a specialist physician, representing redundant work. While potentially important for patient safety, this raises questions of whether an RN triage system results in actual overall system savings. Economic evaluations of $\mathrm{RN}$ and APP roles in specialty care settings have been limited and challenging to interpret, in part due to non-standardised roles and responsibilities across settings. ${ }^{12}$ Second, participating RNs received 4 months of specialist supervision prior to study initiation. This kind of direct supervision is crucial for any successful intervention of this sort and in fact 4 months might not be sufficient. Importantly, the authors note that in the initial 3 months of the study, RNs triaged $75 \%$ of patients to specialists, but at the end of the study period they referred only $45 \%$ of patients. With longitudinal oversight and feedback by specialists, it is plausible that non-physicians could achieve greater confidence in correctly triaging more complex cases. Thus, for programmes such as this to succeed, adequate training is paramount, including comprehensive onboarding for new RNs as well as ongoing easy access to specialist advice and oversight. Third, among the cases triaged to specialists by the RNs, 32\% met at least one of the criteria for specialist oversight; close to $70 \%$ did not meet official criteria for specialist oversight, suggesting that the guidelines might not have adequately captured areas that would be of concern to RNs. Developing and maintaining clear guidelines for non-physician team members with input from end users are key to success.

Paramount to providing quality care is the appropriate use of resources with personnel practising at their full capacity. Programmes such as that described by Donovan et al, ${ }^{10}$ which shift triage decisions from specialty providers to RNs, aim to do just that. But with any such shift comes safety concerns. Donovan $e t$ $a l^{10}$ demonstrate that when considering triage for OSA sleep studies, triage by RNs is not only safe, but potentially even better with respect to guideline adherence. It is also clear that to be successful, such programmes require the development of guideline-driven nonphysician roles, months of direct $\mathrm{RN}$ training and ongoing availability of specialist backup. With continued innovation and fresh thinking about ways to provide care, combined with thoughtful research showing these innovations are safe and effective, we can continue to move closer to the quadruple aim.

Contributors Both authors contributed equally to this work.

Funding The authors have not declared a specific grant for this research from any funding agency in the public, commercial or not-for-profit sectors.

Competing interests DT serves as consultant for BluePath Health on projects related to electronic consultation.

Patient consent for publication Not required. 
Provenance and peer review Commissioned; internally peer reviewed.

\section{ORCID iD}

Delphine S Tuot http://orcid.org/0000-0003-1484-8945

\section{REFERENCES}

1 Liddy C, Moroz I, Mihan A, et al. A systematic review of asynchronous, Provider-to-Provider, electronic consultation services to improve access to specialty care available worldwide. Telemed J E Health 2019;25:184-98.

2 Cook NL, Hicks LS, O'Malley AJ, et al. Access to specialty care and medical services in community health centers. Health Aff 2007;26:1459-68.

3 Forrest CB, Nutting PA, von Schrader S, et al. Primary care physician specialty referral decision making: patient, physician, and health care system determinants. Med Decis Making 2006;26:76-85.

4 Felt-Lisk S, McHugh M, Howell E. Monitoring local safetynet providers: do they have adequate capacity? Health Aff 2002;21:277-83.

5 Vigersky RA, Fish L, Hogan P, et al. The clinical endocrinology workforce: current status and future projections of supply and demand. J Clin Endocrinol Metab 2014;99:3112-21.
6 Singer SJ, Reyes Nieva H, Brede N, et al. Evaluating ambulatory practice safety: the promises project administrators and practice staff surveys. Med Care 2015;53:141-52.

7 Gandhi TK, Sittig DF, Franklin M, et al. Communication breakdown in the outpatient referral process. J Gen Intern Med 2000;15:626-31.

8 Mehrotra A, Forrest CB, Lin CY. Dropping the baton: specialty referrals in the United States. Milbank Q 2011;89:39-68.

9 Tuot DS, Liddy C, Vimalananda VG, et al. Evaluating diverse electronic consultation programs with a common framework. BMC Health Serv Res 2018;18:814.

10 Donovan LM, Palen BN, Syed A. Nurse-Led triage of new sleep referrals is associated with lower risk of potentially contraindicated sleep testing: a retrospective cohort study. BMJ Qual Saf 2021;30:599-607.

11 Antic NA, Buchan C, Esterman A, et al. A randomized controlled trial of nurse-led care for symptomatic moderatesevere obstructive sleep apnea. Am J Respir Crit Care Med 2009;179:501-8.

12 Lopatina E, Donald F, DiCenso A, et al. Economic evaluation of nurse practitioner and clinical nurse specialist roles: a methodological review. Int J Nurs Stud 2017;72:71-82.

13 Martin-Misener R, Harbman P, Donald F, et al. CostEffectiveness of nurse practitioners in primary and specialised ambulatory care: systematic review. BMJ Open 2015;5:e007167. 